\title{
Assessing Cancer Progression and Stable Disease After Neoadjuvant Chemotherapy for Organ-Confined Muscle-Invasive Bladder Cancer
}

\author{
Meera R. Chappidi, M.P.H. ${ }^{1}$, Max Kates, M.D. ${ }^{1}$, Aaron Brant, B.S. ${ }^{1}$, Alexander S. Baras, M.D., \\ Ph.D. ${ }^{2}$, George J. Netto, M.D. ${ }^{2}$, Phillip M. Pierorazio, M.D. ${ }^{1}$, Noah M. Hahn, M.D. ${ }^{3}$, and Trinity \\ J. Bivalacqua, M.D., Ph.D. ${ }^{1}$ \\ ${ }^{1}$ The James Buchanan Brady Urological Institute and Department of Urology, Johns Hopkins, \\ University School of Medicine \\ 2Department of Pathology, Johns Hopkins University School of Medicine \\ ${ }^{3}$ Department of Oncology, Johns Hopkins University School of Medicine
}

\begin{abstract}
Objective-To propose and validate a new approach to stratify clinically staged organ-confined muscle-invasive bladder cancer patients (cT2NOM0) that are pathologic non-responders to neoadjuvant chemotherapy (NAC) to better characterize NAC non-response.
\end{abstract}

Methods-We retrospectively identified radical cystectomy patients with cT2N0M0 disease at our institution (2005-2014) and in the National Cancer Database (NCDB, 2004-2012) for external validation. Patients were stratified as stable (pT2N0M0) or progressors (>pT2 and/or pN+). Primary endpoints were cancer-specific (CSS), overall (OS), and recurrence free survival (RFS).

Results-In the institutional cohort, NAC stable $(\mathrm{n}=17)$ had better OS $(\mathrm{p}=0.05)$ and RFS $(\mathrm{p}=0.04)$ than NAC progressors $(\mathrm{n}=50)$ and comparable OS $(\mathrm{p}=0.7)$ and CSS $(\mathrm{p}=0.09)$ compared to non-NAC stable $(\mathrm{n}=27)$. Multivariable cox proportional hazards models showed larger tumor size (per $\mathrm{cm})$ predicted worse OS (HR=1.20, 95\%CI[1.07-1.35]), CSS (HR=1.27, 95\% CI[1.11$1.45])$, and RFS (HR=1.24, 95\% CI[1.09-1.42]). Similarly, in the NCDB, NAC stable ( $\mathrm{n}=223)$ had improved OS $(\mathrm{p}<0.0001)$ than NAC progressors $(\mathrm{n}=232)$ and comparable $(\mathrm{p}=0.4)$ OS to non-NAC stable $(\mathrm{n}=950)$. Multivariable cox proportional hazards model showed larger tumor size $(\mathrm{HR}=1.03$ per $\mathrm{cm}, 95 \% \mathrm{CI}[1.02,1.03])$ and progression $(\mathrm{HR}=2.69,95 \% \mathrm{CI}[2.40-3.01])$ predicted worse OS.

Conclusions-Distinct survival outcomes suggest NAC non-responders should be further stratified into stable disease and progressors. Comparable survival between non-NAC and NAC stable disease patients suggests NAC stable disease may represent a chemoresistent, but more

Corresponding Author: Meera R. Chappidi, Johns Hopkins University School of Medicine, 733 North Broadway Street, Suite 137, Baltimore, MD 21205, mchappi1@jhmi.edu, Telephone: 410-955-3416, Fax: 410-955-0833.

Publisher's Disclaimer: This is a PDF file of an unedited manuscript that has been accepted for publication. As a service to our customers we are providing this early version of the manuscript. The manuscript will undergo copyediting, typesetting, and review of the resulting proof before it is published in its final citable form. Please note that during the production process errors may be discovered which could affect the content, and all legal disclaimers that apply to the journal pertain.

Disclosures/Conflicts of Interest: None 
indolent phenotype on the disease spectrum. Tumor size is an important prognostic biomarker in NAC non-responders. Clinical predictors of disease progression on NAC were not identified, highlighting the need to explore molecular and genomic subtyping determinants of progression.

\section{Keywords}

urothelial carcinoma; radical cystectomy; neoadjuvant therapy; prognosis

\section{Introduction}

Neoadjuvant chemotherapy (NAC) is commonly used for the treatment of muscle-invasive bladder cancer (MIBC) to improve survival following radical cystectomy (RC). ${ }^{1-3}$

Historically, MIBC patients who receive NAC have been categorized into 3 groups based on RC pathology: "complete responders," "partial responders," or "non-responders." 4-7 Compared to patients who do not receive NAC, a complete or partial response to NAC is associated with improved survival, and non-response to NAC is associated with inferior survival. $^{7}$

Previous studies have stratified NAC non-responders into organ confined vs. non-organ confined based on RC pathology, but these studies do not include pre-NAC clinical TNM classification (cT, cN, cM) in their categorization of non-responders. ${ }^{7-11}$ The importance of clinical classification is demonstrated by the National Comprehensive Cancer Network (NCCN) guidelines, which are widely used to guide clinical practice based on clinical classification. ${ }^{12}$ For clinically classified non-organ confined disease (>cT2 or node positive $[\mathrm{N}+]$ ) the NCCN guidelines "recommend" NAC, but for organ-confined MIBC (cT2N0M0) the guidelines state to "strongly consider" NAC. For this latter group (cT2NOM0), there is less conclusive evidence to support NAC use, so this group could be experiencing either NAC underutilization or over-treatment. Consequently, there is a need for further research to understand the survival implications of NAC in cT2N0M0 patients.

Therefore, we propose stratifying cT2N0M0 patients with NAC non-response into two distinct groups: stable disease $(\mathrm{pT} 2 \mathrm{~N} 0 \mathrm{M} 0=\mathrm{cT} 2 \mathrm{~N} 0 \mathrm{M} 0)$ and pathologic progressors $(\mathrm{pT}>\mathrm{cT} 2$ or $\mathrm{pN}+$ of $\mathrm{pM}+$ pathology). ${ }^{13} \mathrm{In}$ non-NAC cohorts, studies have demonstrated pathologic progression is an independent predictor of worse overall survival (OS), cancer-specific survival (CSS), and recurrence-free survival (RFS) compared to the patients with stable disease. ${ }^{14,15}$ However, it is unknown whether this inferior survival occurs in NAC patients that have pathologic progression compared to stable disease.

Therefore, our objectives in this study were three-fold. First, to further characterize NAC non-response by comparing survival outcomes in clinically classified organ-confined, MIBC patients (cT2N0M0) who received NAC with progression of disease (>pT2 or N+or $\mathrm{M}+$ ) compared to patients who received NAC with stable disease at the time of RC (pT2NOM0). Second, to identify clinical predictors of progression of disease in NAC patients. Third, to compare survival of stable disease patients and progressors between NAC vs. non-NAC patients. 


\section{Materials and Methods}

\section{Patient Cohort}

Our institutional database was queried for patients with cT2N0M0 bladder cancer who underwent RC between 2005-2014. All patients were clinically staged in accordance with NCCN guidelines with transurethral resection of bladder tumor (TURBT) specimen and abdominopelvic and chest imaging. ${ }^{12}$ Pathologic specimens and imaging from outside institutions were re-read by our institution's pathologists and radiologists. Exclusion criteria included patients with indeterminate $\mathrm{pT}$ or $\mathrm{pN}$ classification and non-urothelial primary histology. Patients with primary urothelial histology with squamous $(\mathrm{n}=19)$ or glandular $(n=2)$ differentiation were included. Patients were categorized as having "stable" disease if they had pT2 classification without lymph node involvement (pN0). Patients were categorized as "progressors" if they had >pT2 disease or node positive $(\mathrm{N}+)$ or metastatic disease $(\mathrm{M}+)$ regardless of $\mathrm{pT}$ classification. Patients were further stratified into 4 comparison groups based on progressor status and receipt of NAC; 1) stable disease without NAC (non-NAC stable), 2) disease progression without NAC (non-NAC progressors), 3) stable disease after NAC (NAC stable), 4) disease progression after NAC (NAC progressors).

\section{Tumor Size}

Tumor size was assessed grossly by measuring the greatest dimension of the largest tumor visibly identified during specimen processing. Certainly, variable degrees of scarring can limit the ability to measure tumor size grossly in cases with partial response to NAC, but in our experience this was rare. In cases where tumor was not identified grossly, the bladder lumen and underlying muscular wall was sampled at least twice at the following locations: anterior, posterior, superior, inferior, left, and right.

\section{External Validation Cohort}

Patients with cT2NOM0 urothelial bladder cancer who underwent RC were selected from the National Cancer Database (NCDB) from 2004-2012. The NCDB is a nationwide, joint program of the American Cancer Society and the American College of Surgeons Commission on Cancer, and encompasses $70 \%$ of all newly diagnosed malignancies in the US. ${ }^{16}$ The time from diagnosis to chemotherapy and from diagnosis to RC were used to determine patients that received NAC. We excluded patients without days from diagnosis to chemotherapy, pT classification, vital status, date of last contact, days from diagnosis to RC, patients with discrepancies in $\mathrm{pN}$ classification, and patients who received neoadjuvant nonchemotherapy (radiation, hormone, immunotherapy, or other systemic) treatments. Using the previously described definitions, patients were categorized as non-NAC stable, non-NAC progressors, NAC stable, and NAC progressors.

\section{Survival Statistics}

For the institutional cohort, cause of death was obtained from the National Death Index and recurrence was defined as either local or systemic recurrence. For the NCDB cohort, OS following surgery was determined by subtracting the months between diagnosis to surgery from the months between diagnosis to last follow-up or death. 


\section{Statistical Analyses}

Analysis of variance, Kruskal-Wallis, and $\chi^{2}$ tests were used to compare baseline demographics for continuous and categorical variables as appropriate in both cohorts. Univariable logistic regression was used to determine clinical predictors of disease progression in NAC patients in the institutional cohort.

For survival analyses, Kaplan-Meier (KM) curves were created for each of the 4 comparison groups (non-NAC stable, NAC stable, non-NAC progress, and NAC progressor) to assess the 3 different outcomes of interest: CSS, OS, and RFS. Overall comparisons of KM survival curves were conducted using the Wilcoxon test. If this comparison was significant, pairwise comparisons were made between all groups using the Wilcoxon test. In the NCDB cohort, KM curves were created for OS and both overall and pairwise comparisons between the 4 groups were conducted as described above.

Next, univariable Cox proportional hazard regression models in the institutional cohort were used to determine predictors of CSS, OS, and RFS. For each outcome (CSS, OS, and RFS), a model containing receipt of NAC, progressor status, and the interaction term of these two variables was created. If the interaction term was non-significant, it was not included in multivariable modeling. If progressor status and $\mathrm{pT}$ and/or $\mathrm{pN}$ classification were significant in univariable modeling, only progressor status was used in multivariable models since progressor status was determined by $\mathrm{pT}$ and $\mathrm{pN}$ classification. Subsequently, age at cystectomy and all variables significant on univariable analysis were used in multivariable Cox proportional hazard regression models to determine their association with CSS, OS, and RFS. To validate our multivariable Cox proportion hazards results from the institutional cohort, we created a multivariable model in the NCDB dataset using similar variables. In the NCDB, a sensitivity analysis was conducted including sociodemographic variables with significant differences in distribution between the four groups in the multivariable models. All analyses were conducted using STATA version 12 (StataCorp, College Station, Texas). All reported p-values were 2 sided and p-value $₫ 0.05$ was considered statistically significant for all tests. The Johns Hopkins Hospital (JHH) Institutional Review Board approved this study.

\section{Results}

Of the 762 individuals that underwent RC at our institution from 2005-2014, 39.4\% (300/762) had cT2N0M0 disease and met inclusion criteria. Among the cT2N0M0 patients, there was downstaging (<pT2 and N0) in 61.0\% (105/172) of NAC patients and $20.3 \%$ (26/128) non-NAC patients. Among the 169 non-responders, there were $17(10.1 \%)$ NAC stable, 50 (29.6\%) NAC progressors, 27 (16.0\%) non-NAC stable, and 75 (44.4\%) non-NAC progressors with a median follow-up time of 1.5 years.

In the NCDB, 3,263 patients with cT2N0M0 met inclusion criteria and the traditional definition of non-responders. Among them, there were 223 (6.8\%) NAC stable, 323 (9.9\%) NAC progressors, 950 (29.1\%) non-NAC stable, 1767 (54.1\%) non-NAC progressors with a median follow-up time of 2.8 years. 


\section{JHH Cohort Demographics}

Patients that received NAC had a longer duration from diagnosis of MIBC to cystectomy $(p<0.001)$ compared to non-NAC patients (Table 1a). However, duration from diagnosis of MIBC to cystectomy was comparable in the non-NAC stable group vs. non-NAC progressor group ( $p>0.99$ ) and NAC stable group vs. NAC progressor group $(\mathrm{p}=0.3)$. Tumor size was significantly smaller in the NAC stable group compared to NAC progressors $(\mathrm{p}=0.006)$ and non-NAC progressors $(\mathrm{p}=0.001)$. Of note, tumor size was comparable between the NAC stable and non-NAC stable groups $(\mathrm{p}=0.1)$. All other sociodemographic variables were comparable between groups (all $\mathrm{p}>0.05$ ).

\section{Clinical Predictors of Progression}

Univariable logistic regression did not identify any clinical predictors of disease progression in NAC patients (Table 2).

\section{Five-Year OS, CSS, and RFS}

Five-year CSS, OS, and 2-year RFS for NAC stable, NAC progressors, non-NAC stable, and non-NAC progressors are listed in Figures 1a-c.

\section{NAC Stable vs. NAC Progressive Disease}

OS ( $\mathrm{p}=0.050$ ) and RFS ( $\mathrm{p}=0.04$ ) were significantly worse in NAC progressors compared to NAC stable patients while the worse CSS ( $\mathrm{p}=0.1$ ) in NAC progressors did not meet traditional levels of significance (Figures 1a-c).

\section{Non-NAC Stable vs. non-NAC Progressive Disease}

CSS ( $\mathrm{p}=0.003), \mathrm{OS}(\mathrm{p}=0.02)$, and RFS ( $\mathrm{p}=0.005)$ were all significantly worse in non-NAC progressors compared to non-NAC stable patients (Figures 1a-c).

\section{NAC Stable vs. non-NAC Stable Disease}

RFS ( $\mathrm{p}=0.008$ ) was significantly worse in the NAC stable disease patients compared to the non-NAC stable disease patients while CSS $(\mathrm{p}=0.09)$ and $\mathrm{OS}(\mathrm{p}=0.7)$ were comparable between the two groups (Figures 1a-c).

\section{NAC Progressive vs. non-NAC Progressive Disease}

RFS was significantly worse ( $\mathrm{p}=0.02)$ in NAC progressors compared to non-NAC progressors while the CSS $(\mathrm{p}=0.8)$ and $\mathrm{OS}(\mathrm{p}=0.8)$ were comparable between the two groups (Figures 1a-c).

\section{Cox Proportional Hazards Models}

The multivariable Cox proportional hazard models for CSS, OS, and RFS for all patients (NAC and non-NAC patients) in the institutional cohort are listed in Table 3a. Larger tumor size, presence of LVI, and indeterminate LVI were all predictors of worse CSS, OS, and RFS (Table 3a). 


\section{NCDB Cohort Demographics}

There were significant differences in the distribution of age $(\mathrm{p}<0.001)$, sex $(\mathrm{p}=0.01)$, Charlson score $(\mathrm{p}=0.03)$, tumor size $(\mathrm{p}<0.001)$, days from diagnosis to surgery $(\mathrm{p}<0.001)$, and days from diagnosis to starting NAC ( $\mathrm{p}=0.003$ ) among the groups (Table 1b). All other sociodemographic variables were comparable between groups (all $\mathrm{p}>0.05$ )

\section{NCDB Survival Statistics}

Five-year OS for the NAC stable, NAC progressors, non-NAC stable, and non-NAC progressors was $60.7 \%$ [95\%CI 51.9-68.4\%], 21.4\% [95\%CI 16.1-27.2\%], 64.1\% [95\%CI 60.5-67.5\%], and 28.7\% [95\%CI 26.3-31.1\%] respectively (Figure 1d). The between group comparisons yielded the same qualitative results as in the institutional cohort. OS was significantly improved $(\mathrm{p}<0.0001)$ in the NAC stable disease group vs. NAC progressors, significantly worse $(\mathrm{p}<0.0001)$ in the non-NAC progressors vs. non-NAC stable disease group, comparable $(\mathrm{p}=0.4)$ in the NAC stable vs. non-NAC stable disease groups, and comparable $(\mathrm{p}=0.2)$ in the NAC progressors vs. non-NAC progressor groups.

\section{NCDB Cox-Proportional Hazards Model}

The multivariable cox proportional hazards model for OS in the NCDB cohort for all patients (NAC and non-NAC) is listed in Table 3b. Similar to the JHH cohort, older age, larger tumor size, presence of LVI, and unknown LVI were all predictors of worse OS. Of note, additional predictors of worse OS included increased time from diagnosis to cystectomy $(\mathrm{HR}=1.002$ [95\% CI 1.001, 1.003], $\mathrm{p}<0.001)$ and disease progression $(\mathrm{HR}=2.71$ [95\%CI 2.41, 3.04], $\mathrm{p}<0.001)$. The sensitivity analysis including Charlson score in this multivariable model still showed disease progression ( $\mathrm{HR}=2.69$ [95\% CI 2.40-3.01], $\mathrm{p}<0.001$ ) was the largest predictor of worse OS.

\section{Comment}

Historically, MIBC patients receiving NAC have been categorized as complete responders, partial responders, and non-responders upon RC. However, based on the differences in OS and RFS between NAC progressors and stable disease identified in this study, we propose further subdivision of non-responders into the stable and progressor sub-categories. Indeed the dictum 'NAC non-responders would have been better off had they not gotten NAC at all', does not hold true with stable disease-evidenced by similar OS and CSS in the NAC and non-NAC stable disease groups. This suggests NAC stable disease may represent a chemoresistant but more indolent disease phenotype than NAC progressors. Moreover, we demonstrate tumor size is an prognostic indicator of OS, CSS, and RFS suggesting there is more to NAC response than pathologic downstaging of disease.

To date, this is the first study to demonstrate among NAC non-responders, NAC stable disease has improved OS and RFS than NAC progressors. This was identified both at a tertiary care center, where $96 \%$ (64/67) of patients received platinum-based chemotherapy regimens, as well as in the NCDB which reflects the broader US population with likely more diversity in chemotherapy regimens. Specifically, the NCDB cohort showed after 
adjustment, NAC progressors have a 2.7 times increased risk of all-cause death compared to NAC stable disease.

While the results of this study do not change the current treatment paradigm for bladder cancer, they do provide important insights for new diagnostic tests, neoadjuvant therapies, and adjuvant therapies being developed. As contemporary research efforts focus on utilizing pathologic features and biomarkers to identify individuals that would be responders vs. nonresponders pre-NAC administration, this study suggests that this Manichean view of chemotherapy response while still important is likely too simplistic. ${ }^{17-21}$ Moreover, the difference in OS between NAC stable vs. NAC progressors is especially relevant for the design of clinical trials for adjuvant therapies in NAC patients. Unbalanced enrollment of NAC progressors in one arm of an adjuvant therapy trial could bias results towards improved survival for the other arm. Therefore, in adjuvant therapy clinical trials this bias should be accounted for through either stratification or controlling for progressor status in multivariable modeling.

This study was unable to identify clinical predictors of NAC progression. Therefore, additional efforts should also be focused on developing molecular biomarkers and genomic subtyping of TURBT specimens prior to administration of NAC and RC in order to identify patients who are likely to be NAC progressors. Once created, these tests could supplement clinical criteria to improve the current risk-based approach to NAC administration. ${ }^{22,23}$ Additionally, identifying patients who are not only chemoresistant (both NAC stable and progressors), but will also have disease progression during NAC administration would aid in directing this high-risk cohort to clinical trials and alternative treatment modalities such as neoadjuvant immunotherapies or upfront cystectomy.

This study and the risk-based clinical criteria approach to NAC administration highlight the value of clinically staging bladder cancer to better understand prognosis following RC. However, there is a need for improvement of clinical staging modalities as many patients have upstaging of disease in the pathologic specimen. ${ }^{24,25}$ Recently, new radiographic modalities including dynamic gadolinium and diffusion weighted magnetic resonance imaging have been studied in an effort to increase clinical staging accuracy. ${ }^{26,27}$ Future studies are necessary to understand the potential utility of incorporating these new radiographic measurements to improve the current risk-based approach to NAC administration.

The similar survival outcomes between NAC stable disease and non-NAC stable disease in both JHH and NCDB cohorts has not been identified in prior studies that group together all non-responders. ${ }^{6,7}$ While NAC stable disease individuals were more likely to have disease recurrence than non-NAC stable disease individuals, this increase in disease recurrence did not translate to worse OS or CSS. This suggests there may be something unique about the nature of tumors that are stable in response to NAC that is indicative of a more aggressive but indolent biologic phenotype. However, the comparable OS could also be explained by a selection bias leading to overall healthier patients being selected to receive NAC. Therefore, this finding should be further investigated as a secondary outcome in future randomized 
clinical trials or retrospective analyses of previous randomized clinical trials of NAC for MIBC.

Previous studies on NAC response have focused on the $\mathrm{pT}$ and $\mathrm{pN}$ classification of disease following NAC as the primary prognostic factor. ${ }^{8,28,29}$ However, we demonstrate tumor size is also an important prognostic factor of CSS, OS, and RFS in NAC non-responders. These results imply that while pathologic downstaging is a central aspect of the NAC response, one must also recognize an important role of NAC is likely decreasing tumor size. This tumor size reduction may represent an immediate pharmacodynamics biomarker to document the beneficial systemic effect of NAC on microscopic metastatic disease. Understanding that NAC response can be quantified by both tumor downstaging and tumor size could help provide more accurate prognostic information to patients. These findings warrant future studies with larger sample sizes to better understand the importance of tumor size and its role in the prognosis of NAC stable disease.

This study has several limitations. First, this is an observational, retrospective study by design. Moreover, there is a relatively short median follow-up time for this study. However, prior studies have demonstrated median time to recurrence in MIBC patients undergoing RC is 12 months, and thus the majority of patients who will recur have done so during our follow-up time period. ${ }^{30}$ Additionally, the relatively small sample size with a small number of events limits the power of the study to show statistical differences. Moreover, the data used for this study is from a single, tertiary care center, which can limit the generalizability of findings to other centers that may have different practice patterns. However, the use of the NCDB cohort to validate our findings for OS shows that our findings are likely generalizable to most bladder cancer patients treated at cancer centers. In addition, previous studies have highlighted the potential inaccuracy of clinical classification as many patients are upstaged at the time of RC. ${ }^{24}$ Therefore, it is plausible the progressor group represents patients that were clinically understaged. However, it is equally plausible upstaged patients represent patients that had disease progression. In either case, it is important to realize that while clinical classification may be inaccurate it is the only data available for clinicians to help guide patients in the treatment decision-making process as outlined by the NCCN guidelines. ${ }^{12}$

\section{Conclusions}

In conclusion, this study demonstrates there are two distinct subtypes of pathologic nonresponders to NAC for MIBC: stable disease and progressors. Our inability to identify clinical predictors of NAC progression highlights the need to identify molecular and genomic subtyping determinants of NAC progression, instead of NAC non-response, in the pre-chemotherapy naïve TURBT specimens. Moreover, the therapeutic benefit of NAC may be due to both pathologic downstaging of disease and a decrease in tumor size. Future investigations to understand and better characterize the role of NAC must consider these factors when reporting outcomes of surgical cohorts and adjuvant therapy trials.

\section{Acknowledgments}

Funding: This work was supported in part by the National Institutes of Health [grant number TL1TR001078]. 


\section{References}

1. Grossman HB, Natale RB, Tangen CM, et al. Neoadjuvant chemotherapy plus cystectomy compared with cystectomy alone for locally advanced bladder cancer. N Engl J Med. 2003; 349(9):859-866. [PubMed: 12944571]

2. Advanced Bladder Cancer Meta-analysis Collaboration. Neoadjuvant chemotherapy in invasive bladder cancer: A systematic review and meta-analysis. Lancet. 2003; 361(9373):1927-1934. [PubMed: 12801735]

3. Advanced Bladder Cancer Overview Collaboration. Neoadjuvant chemotherapy for invasive bladder cancer. Cochrane Database Syst Rev. 2005; 2(2) CD005246.

4. Soloway MS. Selecting initial therapy for bladder cancer. Cancer. 1987; 60(3 Suppl):502-513. [PubMed: 3109725]

5. Sternberg CN, de Mulder P, Schornagel JH, et al. Seven year update of an EORTC phase III trial of high-dose intensity M-VAC chemotherapy and G-CSF versus classic M-VAC in advanced urothelial tract tumours. Eur J Cancer. 2006; 42(1):50-54. [PubMed: 16330205]

6. Sonpavde G, Goldman BH, Speights VO, et al. Quality of pathologic response and surgery correlate with survival for patients with completely resected bladder cancer after neoadjuvant chemotherapy. Cancer. 2009; 115(18):4104-4109. [PubMed: 19517476]

7. Gandhi NM, Baras A, Munari E, et al. Gemcitabine and cisplatin neoadjuvant chemotherapy for muscle-invasive urothelial carcinoma: Predicting response and assessing outcomes. Urol Oncol. 2015; 33(5):204.e1-204.e7.

8. Scosyrev E, Messing EM, van Wijngaarden E, et al. Neoadjuvant gemcitabine and cisplatin chemotherapy for locally advanced urothelial cancer of the bladder. Cancer. 2012; 118(1):72-81. [PubMed: 21720989]

9. Siefker-Radtke AO, Dinney CP, Shen Y, et al. A phase 2 clinical trial of sequential neoadjuvant chemotherapy with ifosfamide, doxorubicin, and gemcitabine followed by cisplatin, gemcitabine, and ifosfamide in locally advanced urothelial cancer: Final results. Cancer. 2013; 119(3):540-547. [PubMed: 22914978]

10. El-Gehani F, North S, Ghosh S, Venner P. Improving the outcome of patients with muscle invasive urothelial carcinoma of the bladder with neoadjuvant gemcitabine/cisplatin chemotherapy: A single institution experience. Can Urol Assoc J. 2014; 8(3-4):e287-e293. [PubMed: 24839503]

11. Sobin, LH., Gospodarowicz, MK., Wittekind, C. TNM classification of malignant tumors. 7th. Hoboken, NJ: John Wiley \& Sons, Inc; 2009.

12. National Comprehensive Cancer Network Bladder Cancer Panel. [Accessed 12/10, 2015] NCCN clinical practice guidelines in Oncology Bladder cancer version 2.2015. http://www.nccn.org/ professionals/physician_gls/PDF/bladder.pdf. Updated 2015.

13. Mossanen M, Lee F, Cheng H, et al. Nonresponse to neoadjuvant chemotherapy for muscleinvasive urothelial cell carcinoma of the bladder. Clin Genitourin Cancer. 2014; 12(3):210-213. [PubMed: 24361053]

14. Shariat SF, Palapattu GS, Karakiewicz PI, et al. Discrepancy between clinical and pathologic stage: Impact on prognosis after radical cystectomy. Eur Urol. 2007; 51(1):137-149. discussion 149-51. [PubMed: 16793197]

15. Turker P, Bostrom PJ, Wroclawski ML, et al. Upstaging of urothelial cancer at the time of radical cystectomy: Factors associated with upstaging and its effect on outcome. BJU Int. 2012; 110(6): 804-811. [PubMed: 22321341]

16. Getting started with the 2013 PUF. [Accessed 03/25, 2016] American College of Surgeons Web site. http://ncdbpuf.facs.org/node/274. Updated 2015.

17. Choueiri TK, Jacobus S, Bellmunt J, et al. Neoadjuvant dose-dense methotrexate, vinblastine, doxorubicin, and cisplatin with pegfilgrastim support in muscle-invasive urothelial cancer: Pathologic, radiologic, and biomarker correlates. J Clin Oncol. 2014; 32(18):1889-1894. [PubMed: 24821883]

18. Hemdan T, Malmstrom PU, Jahnson S, Segersten U. Emmprin expression predicts response and survival following cisplatin containing chemotherapy: A validation study in patients with bladder 
cancer from 2 randomized studies of cystectomy with and without neoadjuvant chemotherapy. $\mathbf{J}$ Urol. 2015

19. Plimack ER, Hoffman-Censits JH, Viterbo R, et al. Accelerated methotrexate, vinblastine, doxorubicin, and cisplatin is safe, effective, and efficient neoadjuvant treatment for muscleinvasive bladder cancer: Results of a multicenter phase II study with molecular correlates of response and toxicity. J Clin Oncol. 2014; 32(18):1895-1901. [PubMed: 24821881]

20. McConkey DJ, Choi W, Shen Y, et al. A prognostic gene expression signature in the molecular classification of chemotherapy-naive urothelial cancer is predictive of clinical outcomes from neoadjuvant chemotherapy: A phase 2 trial of dose-dense methotrexate, vinblastine, doxorubicin, and cisplatin with bevacizumab in urothelial cancer. Eur Urol. 2016; 69(5):855-862. [PubMed: 26343003]

21. Lerner SP, McConkey DJ, Hoadley KA, et al. Bladder cancer molecular taxonomy: Summary from a consensus meeting. B1 Cancer. 2016; 2(1):37-47. [PubMed: 27376123]

22. Culp SH, Dickstein RJ, Grossman HB, et al. Refining patient selection for neoadjuvant chemotherapy before radical cystectomy. J Urol. 2014; 191(1):40-47. [PubMed: 23911605]

23. Dickstein RJ, Kamat AM. Contemporary management of locally invasive bladder cancer. Oncology (Williston Park). 2011; 25(14):1396-1405. [PubMed: 22329191]

24. Rais-Bahrami S, Pietryga JA, Nix JW. Contemporary role of advanced imaging for bladder cancer staging. Urol Oncol. 2015

25. Bostrom PJ, van Rhijn BWG, Fleshner NE, et al. Staging and staging errors in bladder cancer. Eur Urol Suppl. 2010; 9:2.

26. Daneshmand S, Ahmadi H, Huynh LN, Dobos N. Preoperative staging of invasive bladder cancer with dynamic gadolinium-enhanced magnetic resonance imaging: Results from a prospective study. Urology. 2012; 80(6):1313-1318. [PubMed: 23040723]

27. Giannarini G, Petralia G, Thoeny HC. Potential and limitations of diffusion-weighted magnetic resonance imaging in kidney, prostate, and bladder cancer including pelvic lymph node staging: A critical analysis of the literature. Eur Urol. 2012; 61(2):326-340. [PubMed: 22000497]

28. Dash A, Pettus JA 4th, Herr HW, et al. A role for neoadjuvant gemcitabine plus cisplatin in muscle-invasive urothelial carcinoma of the bladder: A retrospective experience. Cancer. 2008; 113(9):2471-2477. [PubMed: 18823036]

29. Teramukai S, Nishiyama H, Matsui Y, Ogawa O, Fukushima M. Evaluation for surrogacy of end points by using data from observational studies: Tumor downstaging for evaluating neoadjuvant chemotherapy in invasive bladder cancer. Clin Cancer Res. 2006; 12(1):139-143. [PubMed: 16397035]

30. Stein JP, Lieskovsky G, Cote R, et al. Radical cystectomy in the treatment of invasive bladder cancer: Long-term results in 1,054 patients. J Clin Oncol. 2001; 19(3):666-675. [PubMed: 11157016] 


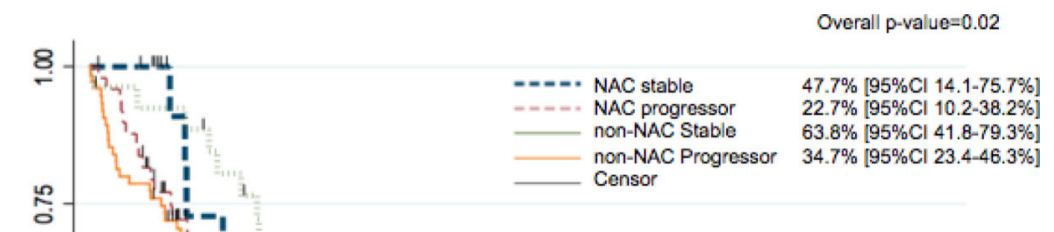

$\begin{array}{lrrrrrl}\text { Number At Risk } & 17 & 6 & 1 & 0 & 0 & 0 \\ \text { NAC Stable } & 17 & 12 & 4 & 2 & 0 & 0 \\ \text { NAC Progressor } & 50 & 16 & 14 & 12 & 5 & 0 \\ \text { Non-NAC Stable } & 27 & 32 & 19 & 12 & 5 & 0\end{array}$

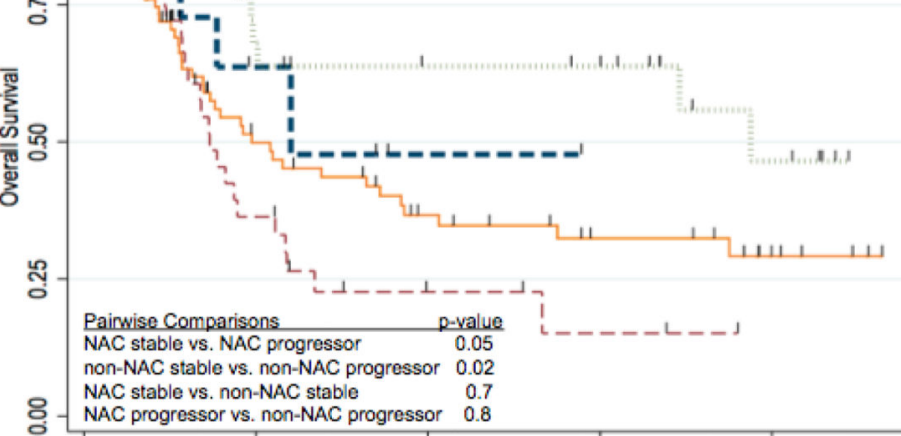

C progresser 0.8

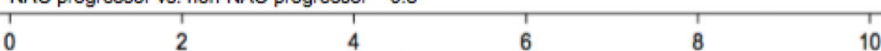

me Since RC (years)
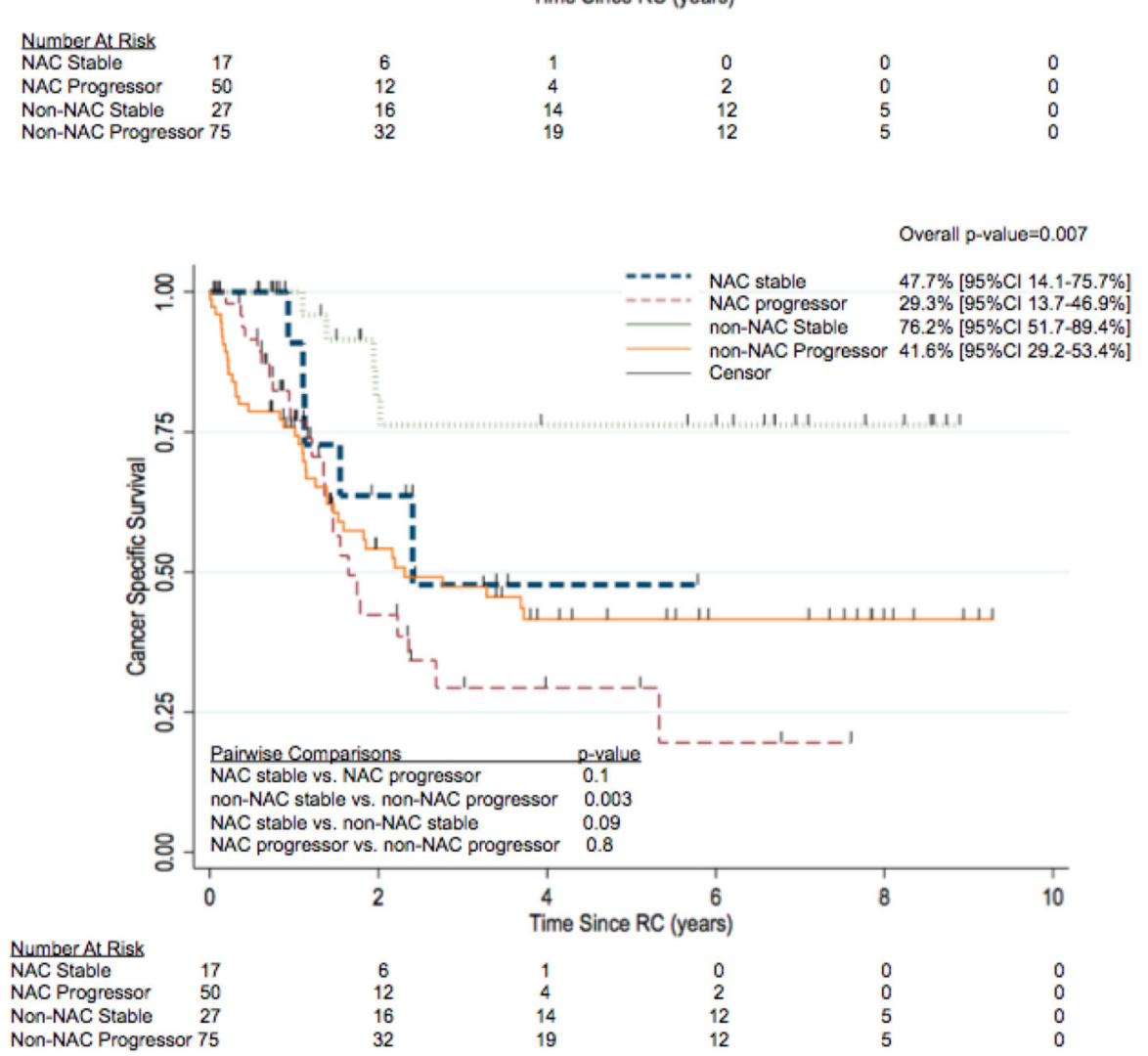

Urology. Author manuscript; available in PMC 2018 April 01. 

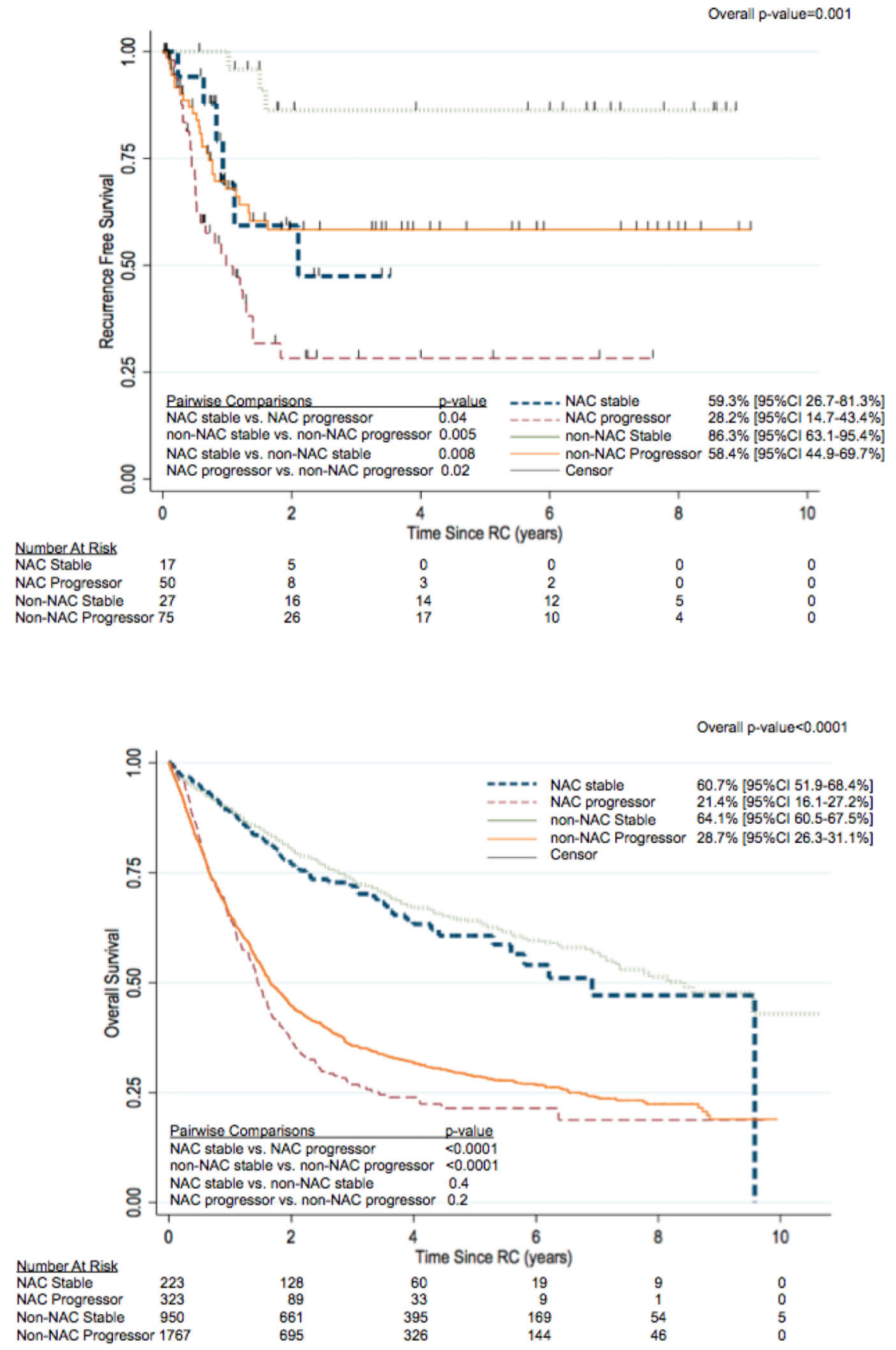

Figure 1.

a. Overall Survival (OS) stratified by NAC and progressor status in institutional cohort.

b. Cancer-Specific Survival (CSS) stratified by NAC and progressor status in the institutional cohort.

c. Recurrence Free Survival (RFS) stratified by NAC and progressor status in the institutional cohort. 
d. Overall Survival (OS) stratified by NAC and progressor status in the National Cancer Database cohort. 


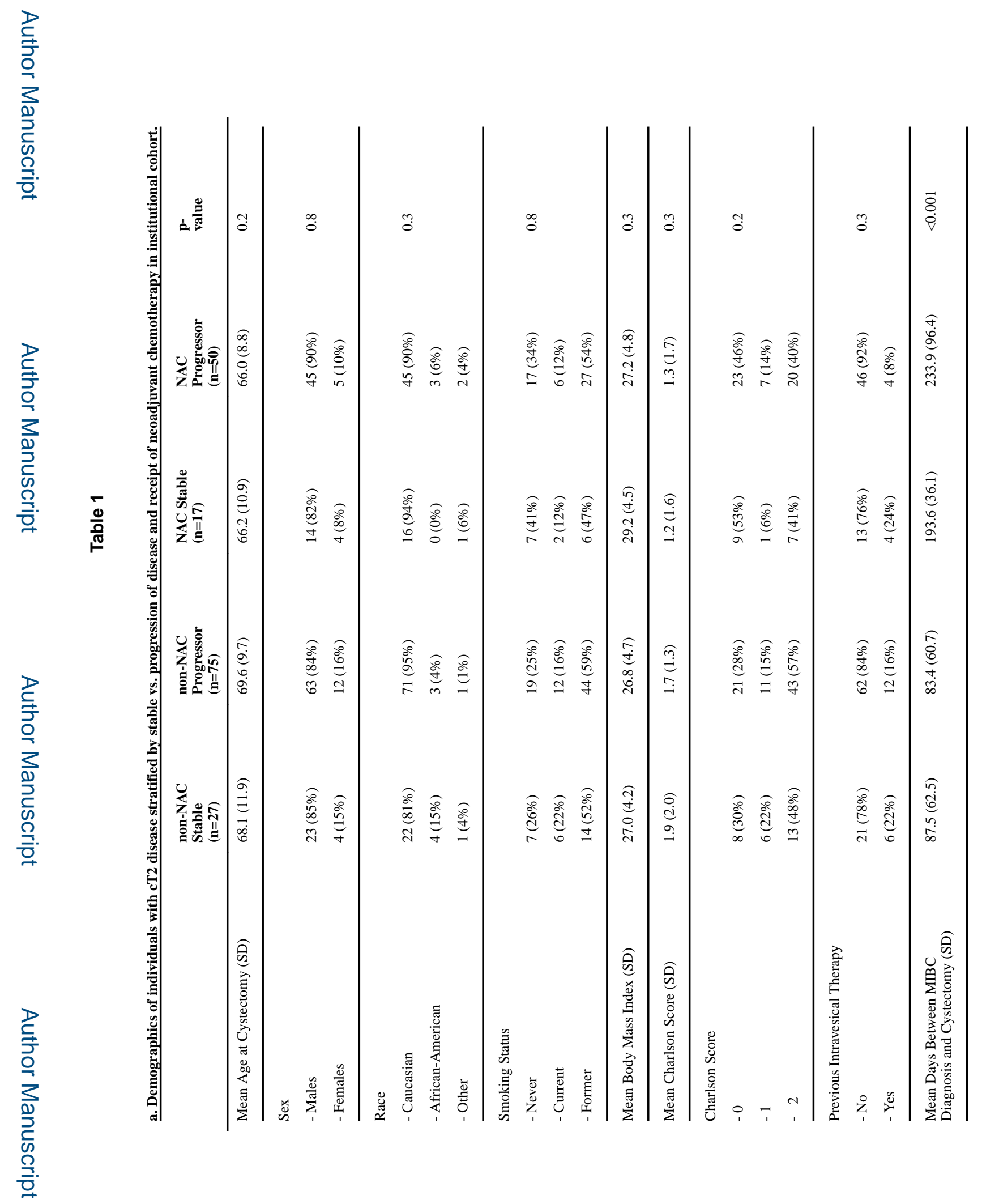

Urology. Author manuscript; available in PMC 2018 April 01. 
Chappidi et al.

Page 15

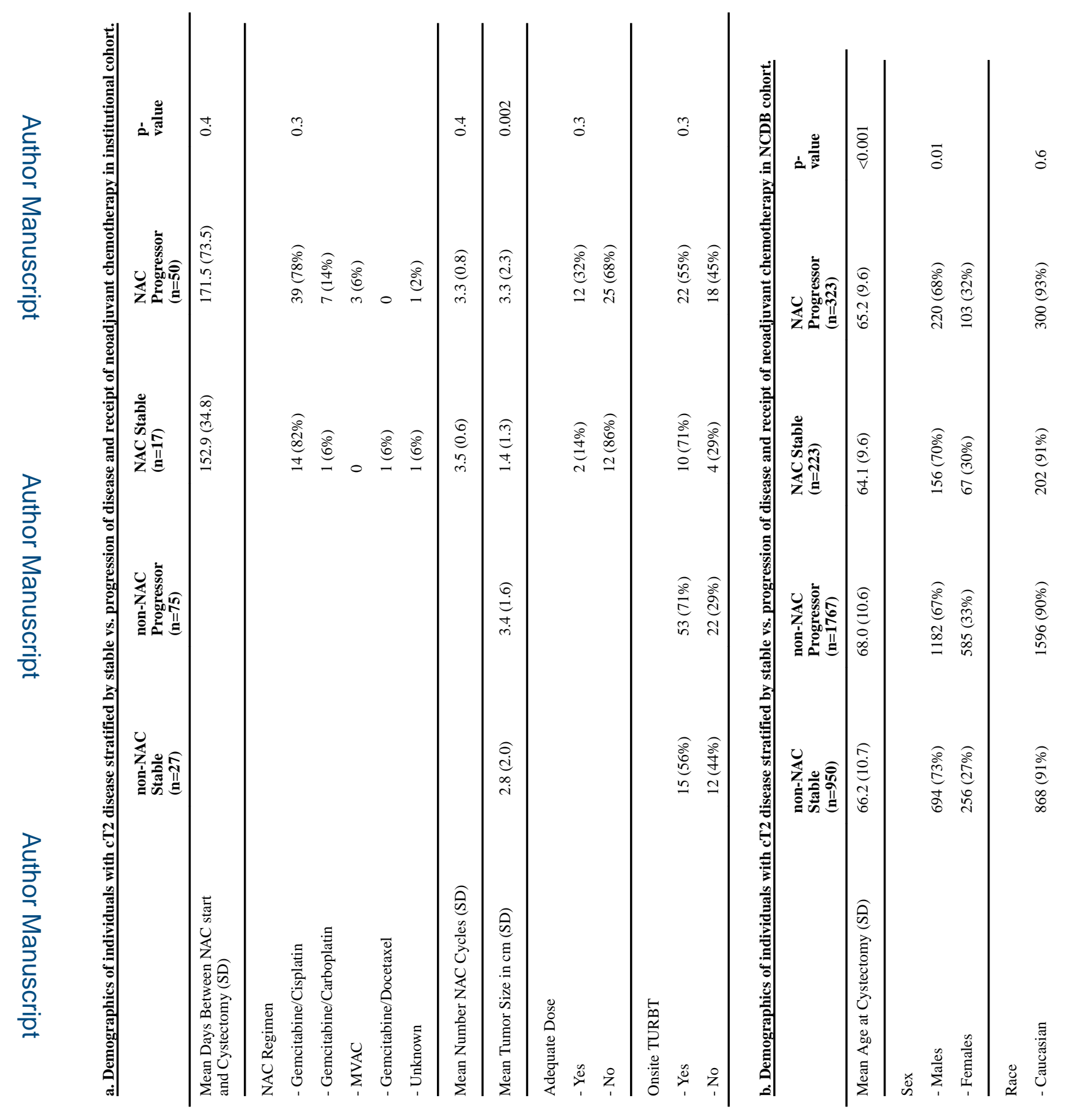

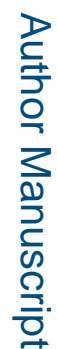

Urology. Author manuscript; available in PMC 2018 April 01. 
Chappidi et al.

Page 16

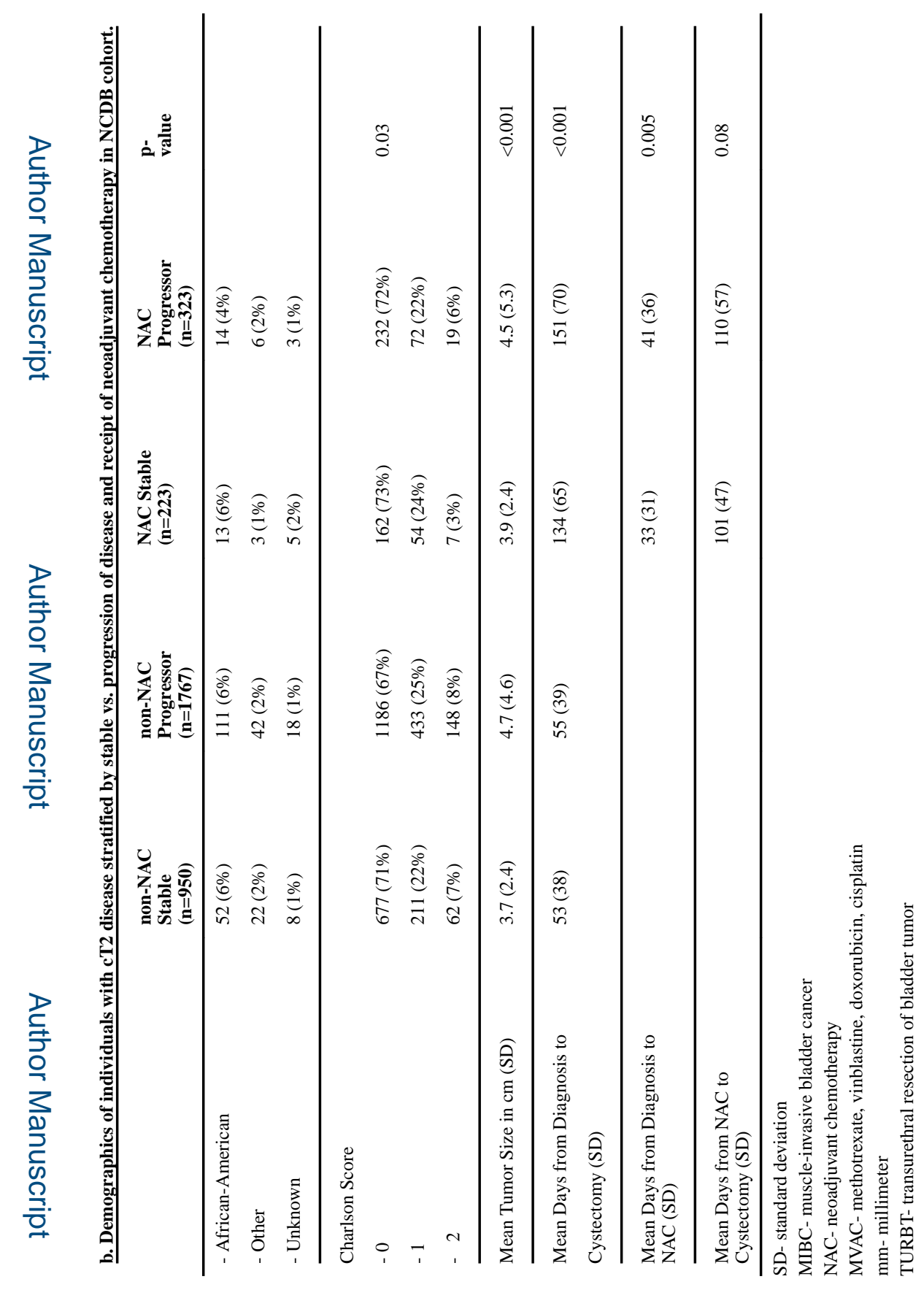

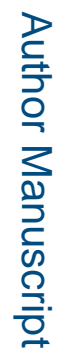

Urology. Author manuscript; available in PMC 2018 April 01. 
Table 2

Univariable logistic regression to determine predictors of progression in NAC patients in institutional cohort.

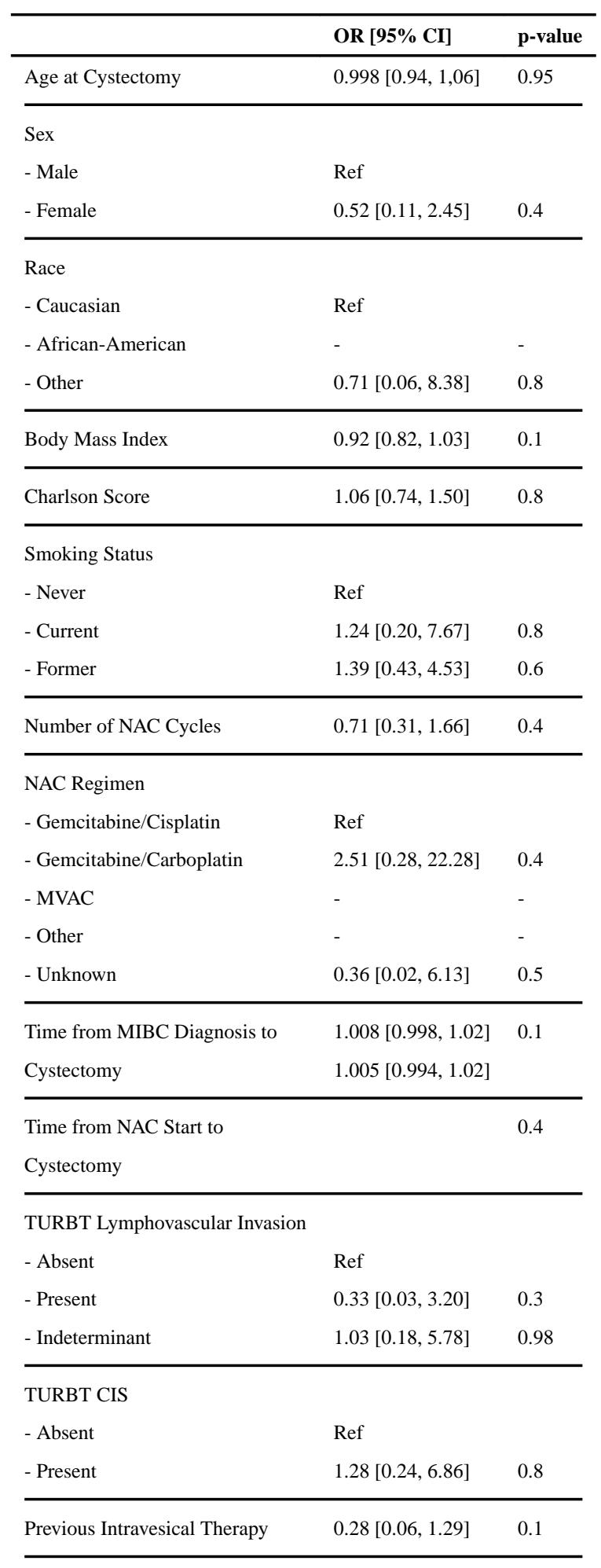

Urology. Author manuscript; available in PMC 2018 April 01. 


\begin{tabular}{lll}
\hline & OR [95\% CI $]$ & p-value \\
\hline Adequate Dose & Ref & \\
- No & $0.35[0.07,1.80]$ & 0.2 \\
- Yes & & \\
\hline Onsite TURBT & Ref & \\
- No & $2.05[0.55,7.63]$ & 0.3 \\
- Yes & & \\
\hline Year of Surgery & Ref & \\
- 2005-09 & $0.55[0.14,2.22]$ & 0.4 \\
- 2010-14 & & \\
\hline Year of MIBC Diagnosis & Ref & \\
- 2004-09 & $0.46[0.11,1.81]$ & 0.3 \\
\hline
\end{tabular}

NAC- neoadjuvant chemotherapy

MVAC- methotrexate, vinblastine, doxorubicin, cispl

MIBC- muscle-invasive bladder cancer

TURBT- transurethral resection of bladder tumor

CIS- carcinoma in situ 


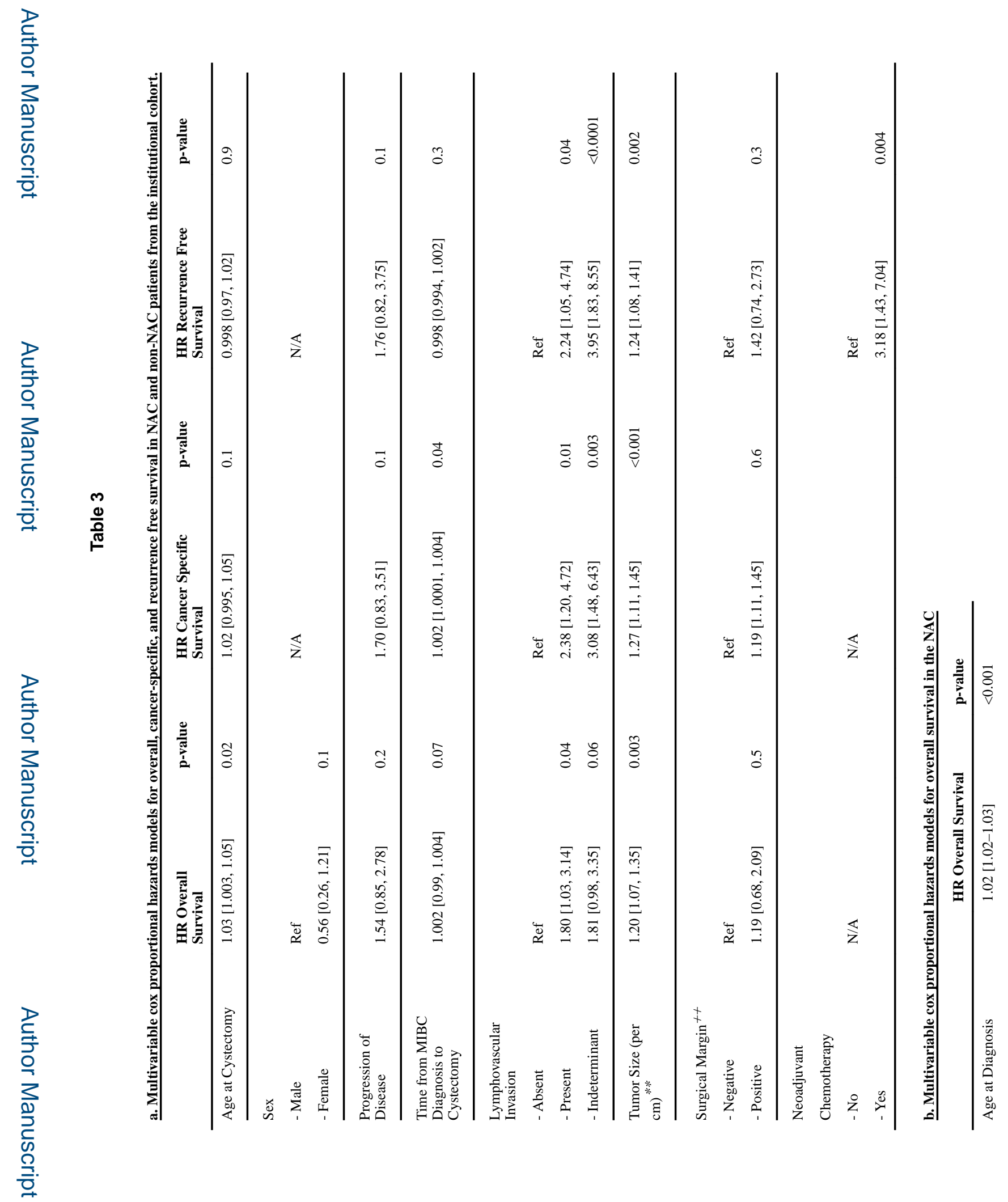

Urology. Author manuscript; available in PMC 2018 April 01. 


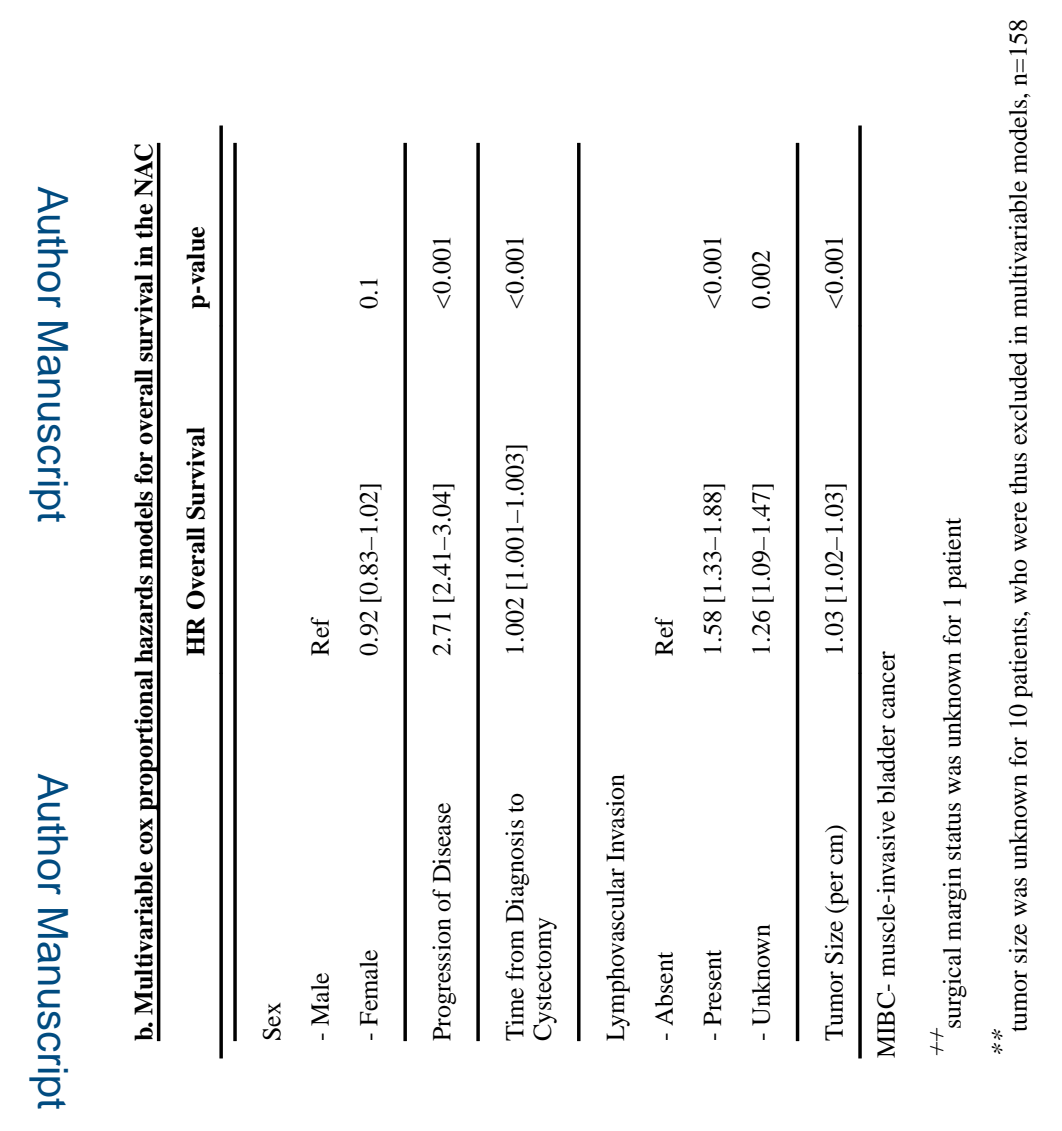

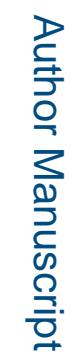

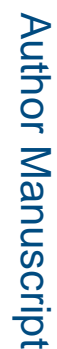

Urology. Author manuscript; available in PMC 2018 April 01. 